\title{
Anopheles gambiae sensu stricto Aquatic Stages Development Comparison between Insectary and Semifield Structure
}

\author{
Eliningaya J. Kweka, ${ }^{1,2,3}$ Filemoni Tenu, ${ }^{3,4}$ Frank Magogo, ${ }^{3,4}$ and Leonard E. G. Mboera ${ }^{3,5}$ \\ ${ }^{1}$ Division of Livestock and Human Health Disease Vector Control, Tropical Pesticides Research Institute, Mosquito Section, \\ P.O. Box 3024, Arusha, Tanzania \\ ${ }^{2}$ Department of Medical Parasitology and Entomology, School of Medicine, Catholic University of Health and Allied Sciences, \\ P.O. Box 1464, Mwanza, Tanzania \\ ${ }^{3}$ Pan African Mosquito Control Association (PAMCA), P.O. Box 9653, Dar es Salaam, Tanzania \\ ${ }^{4}$ National Institute for Medical Research, Amani Medical Research Centre, P.O. Box 81, Muheza, Tanzania \\ ${ }^{5}$ National Institute for Medical Research, Headquarters, P.O. Box 9653, Dar es Salaam, Tanzania \\ Correspondence should be addressed to Eliningaya J. Kweka; pat.kweka@gmail.com
}

Received 18 September 2014; Revised 10 December 2014; Accepted 10 December 2014

Academic Editor: Cleber Galvão

Copyright (c) 2015 Eliningaya J. Kweka et al. This is an open access article distributed under the Creative Commons Attribution License, which permits unrestricted use, distribution, and reproduction in any medium, provided the original work is properly cited.

\begin{abstract}
Effective malaria vector control initiatives need a clear understanding of mosquito behaviour and its ecology. This study compared larvae development to adult emergence in insectary and malaria-sphere (SFS). This is the preliminary study which gives an insight to forthcoming studies. Anopheles gambiae sensu stricto eggs were hatched in insectary and transferred in densities of 20 and 50 per microhabitat with twenty replicates of each density. Both densities of larvae were reared in semifield structure and in insectary from the same batch of eggs. They were provided with tetramin fish food. In both densities of 20 and 50, pupation rate and time were found to be similar in SFS and insectary, but, in survivorship from larvae to pupae at density of 50, more larvae survived significantly to pupae stage in SFS than in insectary $(P=0.002)$. The adult emergence rates were similar for densities of 20 and 50 between SFS and Insectary. There was a significant difference between SFS and insectary in light intensity $(P=0.001)$ and temperatures $(P=0.001)$, with SFS having higher rates than insectary. The findings of this study have shown that larvae development rates are encouraging having semifield structures for malaria vector rearing for behavioural studies toward malaria control.
\end{abstract}

\section{Background}

Currently, malaria vectors control activities have succeeded in shrinking vector distribution map in different areas across Africa [1-3]. Malaria vector control programmes mainly involve insecticides treated nets and indoor residual spray programmes of which most are pyrethroid based [4-6]. The two methods have suffered major drawbacks due to mushrooming of pyrethroid resistance within vector populations [6-10] and vectors house entry behavioural changes [3]. Additional techniques such as larvae source management strategy [11-13] and mass trapping of adult vector outdoors $[14,15]$ have added value but not to zero vector population or malaria transmission.

Several behavioural studies such as trial of novel pesticides, repellent, fungicides, and treated materials need high number of mosquitoes from insectary to serve as control. In other studies, sterile insect technique (SIT), which is regarded to be environmentally safe for disease vectors control and very practical in isolated areas such as islands, needs very high number of mosquitoes for it to be realised [16]. In El Salvador, a range of 0.5 to 1.25 million sterile male pupae were released on daily bases from 1977 to 1979 during the SIT programme against Anopheles albimanus [17-19]. A million sterile males of An. arabiensis were released per day for one year, in Sudan [20]. This technique has been very impressive in Tsetse fly elimination in Zanzibar [21]. In all SIT trials, the success of the programme depends on massive release of sterile males. To achieve and maintain massive release of adults, mass rearing infrastructure which ensures high survivorship of the larvae to adult at sustainable and affordable production in African setting is therefore required. The immature stages 
developmental rate quality is of significant importance in influencing emerging adults fitness $[22,23]$ and has adverse effect on adult survivorship, mating, host seeking, and flight ability $[22,24,25]$.

Therefore, it was the aim of this study to investigate the relevance of using SFS compared to insectary rearing of Anopheles gambiae s.s. by measuring survivorship from larvae to pupae, pupation, adult emergence rates, light intensity, and temperature.

\section{Method}

2.1. Description of Insectary and SFS. This study was carried out in Muheza (S05.17041; E038.77873, 216M) using the mosquitoes insectary of National Institute for Medical Research (NIMR), Amani Medical Research Centre. The larvae rearing room has dimensions of $5.5 \mathrm{~m}$ length, $3.0 \mathrm{~m}$ width, and $3.0 \mathrm{~m}$ height in an insectary with total dimensions of $11.6 \mathrm{~m}$ length and $10.9 \mathrm{~m}$ width. The larvae rearing room is maintained at temperature of $28 \pm 2$ degrees Celsius and relative humidity of $78 \pm 2 \%$. The photoperiod is $12 \mathrm{~L}: 12 \mathrm{D}$. The temperature, relative humidity, and light in the insectary are maintained using the electric heaters, humidifiers, and bulbs throughout with uncovered window glasses.

The SFS used in this study is located at the same area. The SFS has been constructed to simulate a natural outdoor environment [26]. In SFS vegetation was allowed to grow as in natural environment with a small hut inside $(2.74 \times 2.74 \times$ $1.83 \mathrm{~m})$. SFS has dimensions of $12.2 \mathrm{~m}$ length and $8.2 \mathrm{~m}$ width [26-28]. SFS is covered with strong netting material allowing free wind flow and precipitations to mimic the natural climatic conditions. Photophase in SFS depends on natural sunrise and sunset.

2.2. Mosquito Colony Rearing. Anopheles gambiae s.s. Kisumu strain (R70), which has been colonized since 1992, was used. The colony is susceptible to all kinds of insecticides approved by World Health Organisation Pesticide Evaluation Scheme (WHOPES).

2.3. Rearing of Aquatic Stages. Two rearing systems were used for monitoring the development of aquatic stages of $A n$. gambiae s.s. during this preliminary study. Semifield structure (SFS) and ordinary insectary were compared in this study. Mosquito eggs were hatched and, after one hour, first instars larvae were separated. Experiments had two densities of 20 and 50 larvae per replicate. Each density had 20 replicates reared in each system. Developmental time, pupation rates, and survivorship from larvae to pupae were monitored. Larvae were fed with fish food daily (manufactured by Tetra $\mathrm{GmbH}$, Herrenteich 78, 49324 Melle, expiring on March 2015) at a rate of $0.0003 \mathrm{gm}$ per larvae. The rearing bowls had a diameter of $5.6 \mathrm{~cm}$ and depth of $2.2 \mathrm{~cm}$ to mimic the small and temporal natural breeding habitats.

2.4. Survivorship from Larvae to Pupae and Developmental Monitoring. From hatching day, larvae development was monitored daily by counting the larvae by age structure (i.e., the age of larvae from hatching day to pupae) as described elsewhere $[22,29]$. After the first collected pupae, the pupae collection was done every morning and evening to avoid losing emerging adults $[22,29]$, in both the insectary and the SFS. The pupae collected were placed in a paper cup with some water from their native bowl. Pupae collected were separated by replicates and days.

2.5. Temperature and Light Intensity in Structures. Temperature and light intensity in both structures were recorded by Onset HOBO (Part Number UA-002-64, Patent 6,826,664). The onset HOBO was started at the beginning of experiment and switched off at the end of the experiments.

2.6. Statistical Analysis. Data were recorded in Excel sheet and transferred to PWAS Statistics 18.0 (SPSS Inc., Chicago, IL) for analysis after being cross-checked. The Wilcoxon signed ranks test was used to compare the daily survivorship, pupation, and adult mergence rates between insectary and SFS for densities of 20 and 50. Microsoft Excel sheet was used for drawing the histograms and trend graphs. Comparison was considered significant when $P$ value was below 0.05 .

\section{Results}

3.1. Survivorship from Larvae to Adults. Larvae survivorship to adult was 81.8 and $86.3 \%$ in density of 20 and 90.2 and $81.6 \%$ in density of 50 for SFS and insectary, respectively. The survivorship of larvae at a density of 20 was not statistically significant between insectary and SFS $(P=0.255)$ while in density of 50 the survivorship to adult in SFS was significantly higher than in insectary $(P=0.002)$ (Figure 1$)$. In SFS the survivorship from larvae to adult took 16 to 17 days while in insectary it took 17 to 18 days.

3.2. Pupation Time. In SFS, pupation in density of 50 was between day 8 and day 15 while in the insectary it was between days 6 and 11 (Figure 2). Both pupation rates of 20 and 50 densities were not statistically different between insectary and SFS $(P=0.465$ and $P=0.176$, resp. $)$.

3.3. Adult Emergence Rates. Adult emergence rates in insectary were 78.0 and $84.7 \%$ while for SFS they were 78.5 and 81.8 for densities of 20 and 50, respectively. The emergence rates for insectary and SFS at a density of 20 were not statistically different $(P=0.403)$. For density of $50,84.7 \%$ adults emerged in insectary against $81.8 \%$ in SFS. Thus, there were slightly more adults emerging in the insectary, but this difference was not statistically significant $(P=0.053)$ (Figure 3).

3.4. Temperature and Light Intensity. Temperature and light intensity had great variations between SFS and insectary. The light intensity was significantly higher in SFS than in insectary $(P \geq 0.001)$. Temperature was significantly higher in the insectary installed with electric heaters than in the SFS $(P \geq 0.001)$ (Figure 4). 


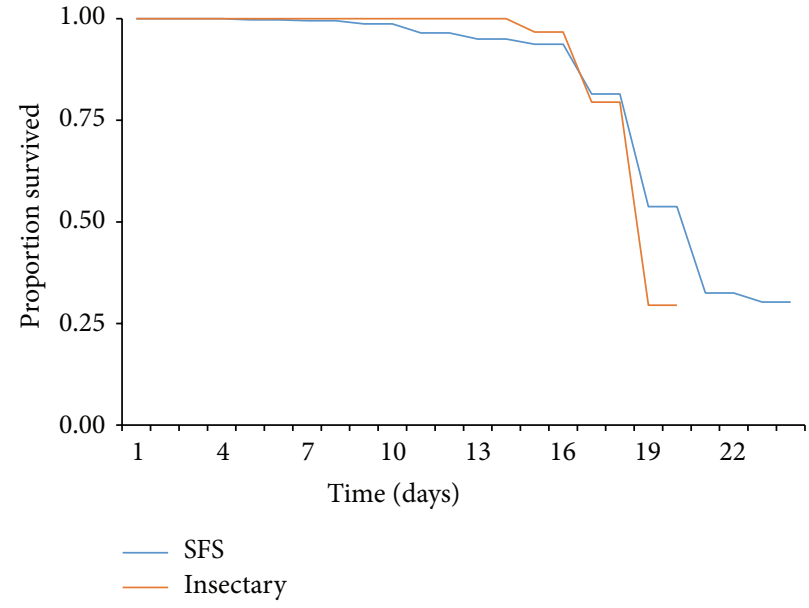

(a)

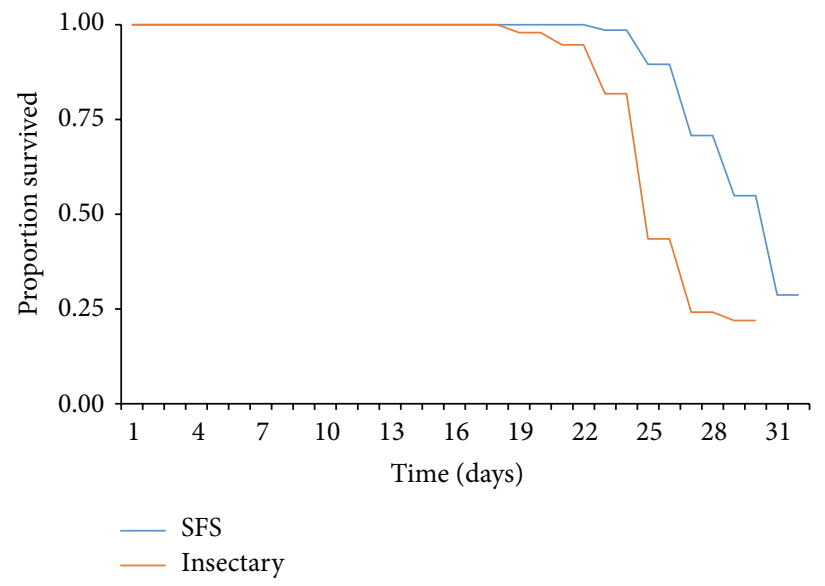

(b)

FIGURE 1: Daily proportion of larvae that survived to adult in densities of 20 (a) and 50 (b) in both insectary and SFS conditions.

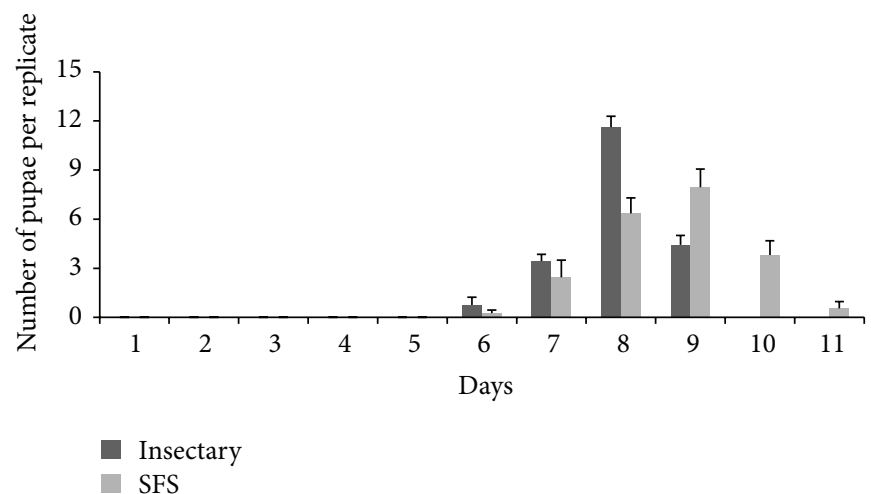

(a)

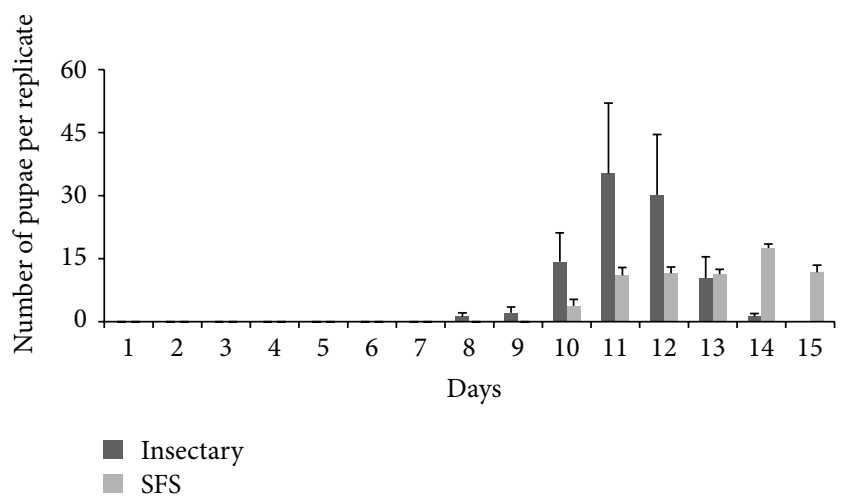

(b)

FIGURE 2: Pupation rates per replicate in SFS and insectary at densities of 20 (a) and 50 (b) during the experimental days (the bars present 95\% Confidence Interval).

\section{Discussion}

The results of this study have shown that mosquitoes rearing in African setting using SFS have more advantages in larval survival and developmental time when compared to the ordinary insectary. The larval survivorship in SFS have been found to convince that, large scale rearing of mosquitoes can be done in SFS than in insectary due to shorter larval development time.

The larval development rates to adult in high larval density in SFS compared to in insectary were unexpected based on the factor that insectary has constant temperature throughout the day $(24 \mathrm{hr}$ ) which has been determined to be the most sensitive function for mosquito larvae development [30-32]. Larvae survivorship and development rely much on food quality and its availability, temperature, and light intensity [33]. Sunlight intensity and temperature are of importance for microbial community development and algal biomass photosynthetic process for food production, hence provision of high quality food for aquatic mosquitoes stages development and survivorship in seminatural ecology [12, 33-35]. In natural ecology, it has been found that An. gambiae

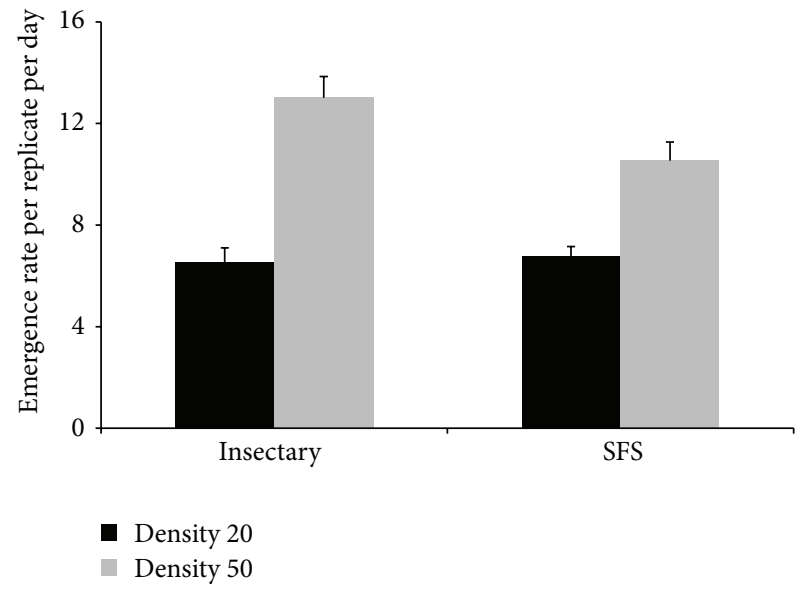

FIGURE 3: The emergence rates of adult mosquitoes in both SFS and insectary for densities of 20 and 50 (the bars present $95 \%$ Confidence Interval).

s.l. prefers open shallow habitats which receives direct sunlit with no vegetation cover [36]. This ensures rapid algae 


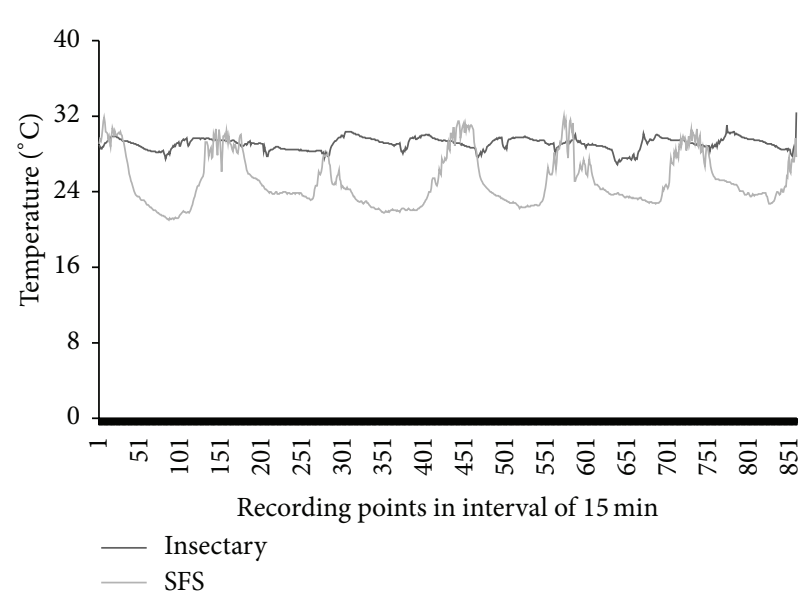

(a)

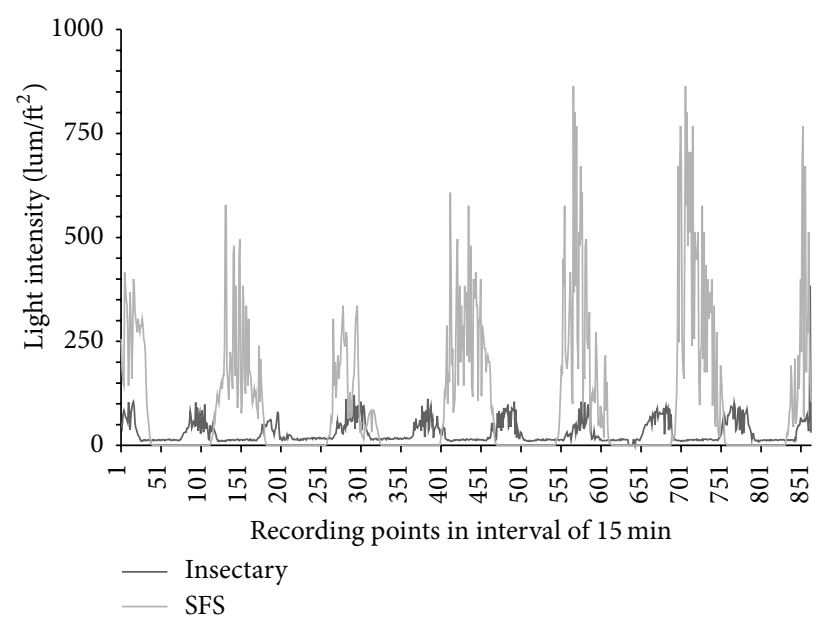

(b)

FIGURE 4: Variations of temperature (a) and light intensity (b) in SFS and insectary for the experimental duration.

biomass and bacterial growth and detritus decomposition, hence abundance food sources for larvae development. Algal biomass has been found to influence larvae abundance and development $[11,12]$.

Pupation time and rate in this study have been observed to be similar for both densities of 20 and 50. This implies that both insectary and SFS have potential of larvae survivorship to pupation at equal rates. In natural population, shortened pupation time has influence on fitness of adult emergence and subsequent biological life cycle including predators avoidance $[22,23]$. The influence of day time temperature in SFS had been attributed to the difference in adult emergence as it has been appreciated to be sensitive in pupae developmental process to adult [30,31]. In spite of this difference, SFS is still of priority in African setting based on the following factors. Firstly, there are the high electricity bills to keep the heaters, humidifiers, and light on in insectary at this era of financial crisis. Secondly, Tanzania has been experiencing frequent power cuts and power rationing. Currently, there is increased insecticide resistance status among malaria vectors globally to common pyrethroids and other classes of insecticides used for public health vectors control $[5,9,10,37,38]$. Alternative methods of vectors control are now urgently needed to complement the control efforts achieved through indoor residual spray (IRS) and insecticides treated nets (ITNs) coverage. The wide coverage of ITNs and IRS programmes has influenced mosquitoes host seeking behavioural changes $[3,4]$. These vector behavioural changes have caused outdoor malaria transmission due to forced exophily and repellence effect of insecticides indoors hence resting and feeding outdoors $[3,4,38]$. Another drawback is the increasing restriction of insecticide usage due to increased mortality of nontargeted organisms, increased toxicity to mammals, and other effects on biological systems [39]. Resistance to most of used insecticides globally and climate changes variability might lead to serious mosquito resurgence and reemerging of mosquitoborne diseases [40-44].
In the light of currently existing mosquitoes control difficulties, alternative methods have to be suggested to complement the existing methods, such as genetic control (use of sterile mosquitoes). This method was previously found to be effective and environmentally safe for insect control that has been successful in controlling mosquitoes, tsetse flies, and agricultural pests $[20,45,46]$. Mosquito mass rearing in African setting is affordable as the current study has shown. Other studies in Kenya [26] and in Ifakara, Tanzania [47], have shown that An. gambiae s.s. and An. arabiensis emerging in the SFS can survive for more than ten generations. Mosquitoes can propagate within the SFS which is an added value for the SIT trials and implementation in African malaria endemic area without much coverage of modern infrastructure to raise insects [47]. SIT application in African setting will be of added value as it is not compromised with insecticides resistance as other tools such as ITNs and IRS. For mosquitoes SIT has shown some promising achievements both in laboratory and in small scale trials $[20,48]$.

Under the scope of this study, mosquito rearing of African malaria vectors, An. gambiae s.l., can be coordinated in SFS with expectations of sufficient mosquitoes production to meet demand for behavioural studies in control programmes.

\section{Conclusion}

This study has found that An. gambiae s.s. rearing can be as much efficient in SFS as in insectary, which in turn reduces the costs for mass rearing of mosquitoes for research or control measures. Further research has to be done on analysing the proportion of male to female adults in higher densities in SFS to facilitate the estimation for large scale production and costs realities.

\section{Conflict of Interests}

The authors declare that they have no conflict of interests in this work. 


\section{Authors' Contribution}

Eliningaya J. Kweka and Leonard E. G. Mboera conceived and designed the study, carried results interpretation, and drafted and revised this paper. Frank Magogo performed experiments and revised this paper. Filemoni Tenu did data analysis and interpretations and revised this paper. All authors agreed upon submission of this paper.

\section{Acknowledgments}

The authors wish to thank the National Institute for Medical Research, Amani Medical Research Centre, for providing insectary and SFS facilities which made this study possible. They thank Thobias Sekanyika, Irene Semkiwa, and Isaya Kibwana for their valuable contribution during experimental setup and Joseph Myamba for his valuable comments during designing of the study. The study was funded by SIDA through TASENE program which funds Eliningaya J. Kweka Postdoctoral training program. This study was granted ethical approval from National Institute for Medical Research Ethics Committee, with research permit NIMR/ HQ/R.8a/Vol.IX/1584. This paper is published with the permission of the Director General of the National Institute for Medical Research.

\section{References}

[1] Y. A. Derua, M. Alifrangis, K. M. Hosea et al., "Change in composition of the Anopheles gambiae complex and its possible implications for the transmission of malaria and lymphatic filariasis in north-eastern Tanzania," Malaria Journal, vol. 11, article 188, 2012.

[2] D. W. Meyrowitsch, E. M. Pedersen, M. Alifrangis et al., "Is the current decline in malaria burden in sub-Saharan Africa due to a decrease in vector population?" Malaria Journal, vol. 10, article 188, 2011.

[3] G. G. Padonou, G. Gbedjissi, A. Yadouleton et al., "Decreased proportions of indoor feeding and endophily in Anopheles gambiae s.l. populations following the indoor residual spraying and insecticide-treated net interventions in Benin (West Africa)," Parasites and Vectors, vol. 5, article 262, 2012.

[4] R. A. Ossè, R. Aïkpon, G. L. Gbédjissi et al., "A shift from Indoor Residual Spraying (IRS) with bendiocarb to Long-Lasting Insecticidal (mosquito) Nets (LLINs) associated with changes in malaria transmission indicators in pyrethroid resistance areas in Benin," Parasites and Vectors, vol. 6, article 73, 2013.

[5] M. Okia, R. Ndyomugyenyi, J. Kirunda et al., "Bioefficacy of long-lasting insecticidal nets against pyrethroid-resistant populations of Anopheles gambiae s.s. from different malaria transmission zones in Uganda," Parasites and Vectors, vol. 6, no. 1, article 130, 2013.

[6] G. F. Killeen, A. Seyoum, C. Sikaala et al., "Eliminating malaria vectors," Parasites and Vectors, vol. 6, article 172, 2013.

[7] Z. S. Brown, K. L. Dickinson, and R. A. Kramer, "Insecticide resistance and malaria vector control: the importance of fitness cost mechanisms in determining economically optimal control trajectories," Journal of Economic Entomology, vol. 106, no. 1, pp. 366-374, 2013.
[8] T. M. Lo and M. Coetzee, "Marked biological differences between insecticide resistant and susceptible strains of Anopheles funestus infected with the murine parasite Plasmodium berghei," Parasites and Vectors, vol. 6, article 184, 2013.

[9] R. Aïkpon, F. Agossa, R. Ossè et al., "Bendiocarb resistance in Anopheles gambiae s.l. populations from Atacora department in Benin, West Africa: a threat for malaria vector control," Parasites and Vectors, vol. 6, article 192, 2013.

[10] N. Protopopoff, J. Matowo, R. Malima et al., "High level of resistance in the mosquito Anopheles gambiae to pyrethroid insecticides and reduced susceptibility to bendiocarb in NorthWestern Tanzania," Malaria Journal, vol. 12, article 149, 2013.

[11] E. J. Kweka, G. Zhou, S. Munga et al., "Anopheline larval habitats seasonality and species distribution: a prerequisite for effective targeted larval habitats control programmes," PLoS ONE, vol. 7, no. 12, Article ID e52084, 2012.

[12] E. J. Kweka, G. Zhou, M.-C. Lee et al., "Evaluation of two methods of estimating larval habitat productivity in Western Kenya highlands," Parasites and Vectors, vol. 4, article 110, 2011.

[13] B. A. Ndenga, J. A. Simbauni, J. P. Mbugi, A. K. Githeko, and U. Fillinger, "Productivity of malaria vectors from different habitat types in the Western Kenya highlands," PLoS ONE, vol. 6, no. 4, Article ID e19473, 2011.

[14] N. S. Matowo, J. Moore, S. Mapua et al., "Using a new odourbaited device to explore options for luring and killing outdoorbiting malaria vectors: a report on design and field evaluation of the Mosquito Landing Box," Parasites and Vectors, vol. 6, article 137, 2013.

[15] N. J. Govella, P. P. Chaki, J. M. Mpangile, and G. F. Killeen, "Monitoring mosquitoes in urban Dar es Salaam: evaluation of resting boxes, window exit traps, CDC light traps, Ifakara tent traps and human landing catches," Parasites and Vectors, vol. 4, article 40, 2011.

[16] E. Knipling, "Possibilities of insect control or eradication through use of sexually sterile males," Journal of Economic Entomology, vol. 48, pp. 459-462, 1955.

[17] D. A. Dame, M. Q. Benedict, A. S. Robinson, and B. G. J. Knols, "Historical applications of induced sterilisation in field populations of mosquitoes," Malaria Journal, vol. 8, supplement 2, article S2, 2009.

[18] D. A. Dame and H. R. Ford, "Chemosterilization and its permanency in mosquitoes," Nature, vol. 201, no. 4920, pp. 733-734, 1964.

[19] R. E. Lowe, D. L. Bailey, and D. A. Dame, "Efficiency of techniques for the mass release of sterile male Anopheles albimanus Wiedemann in El Salvador," The American Journal of Tropical Medicine and Hygiene, vol. 29, no. 4, pp. 695-703, 1980.

[20] A. S. Robinson, G. Franz, and P. W. Atkinson, "Insect transgenesis and its potential role in agriculture and human health," Insect Biochemistry and Molecular Biology, vol. 34, no. 2, pp. 113-120, 2004.

[21] M. J. Vreysen, K. M. Saleh, M. Y. Ali et al., "Glossina austeni (Diptera: Glossinidae) eradicated on the island of Unguja, Zanzibar, using the sterile insect technique," Journal of Economic Entomology, vol. 93, pp. 123-135, 2000.

[22] E. J. Kweka, G. Zhou, L. B. Beilhe et al., "Effects of co-habitation between Anopheles gambiae s.s. and Culex quinquefasciatus aquatic stages on life history traits," Parasites and Vectors, vol. 5, article 33, 2012.

[23] K. R. Ng’habi, B. John, G. Nkwengulila, B. G. J. Knols, G. F. Killeen, and H. M. Ferguson, "Effect of larval crowding on 
mating competitiveness of Anopheles gambiae mosquitoes," Malaria Journal, vol. 4, article 49, 2005.

[24] J. R. Gilles, R. S. Lees, S. M. Soliban, and M. Q. Benedict, "Density-dependent effects in experimental larval populations of Anopheles arabiensis (Diptera: Culicidae) can be negative, neutral, or overcompensatory depending on density and diet levels," Journal of Economic Entomology, vol. 48, pp. 296-304, 2011.

[25] E. O. Lyimo and W. Takken, "Effects of adult body size on fecundity and the pregravid rate of Anopheles gambiae females in Tanzania," Medical \& Veterinary Entomology, vol. 7, no. 4, pp. 328-332, 1993.

[26] B. G. J. Knols, B. N. Njiru, E. M. Mathenge, W. R. Mukabana, J. C. Beier, and G. F. Killeen, "MalariaSphere: a greenhouse-enclosed simulation of a natural Anopheles gambiae (Diptera: Culicidae) ecosystem in western Kenya," Malaria Journal, vol. 1, article 19, 2002.

[27] J. Kitau, H. Pates, T. R. Rwegoshora et al., "The effect of Mosquito Magnet Liberty plus trap on the human mosquito biting rate under semi-field conditions," Journal of the American Mosquito Control Association, vol. 26, no. 3, pp. 287-294, 2010.

[28] W. H. Schmied, W. Takken, G. F. Killeen, B. G. J. Knols, and R. C. Smallegange, "Evaluation of two counterflow traps for testing behaviour-mediating compounds for the malaria vector Anopheles gambiae s.s. under semi-field conditions in Tanzania," Malaria Journal, vol. 7, article 230, 2008.

[29] E. J. Kweka, G. Zhou, T. M. Gilbreath et al., "Predation efficiency of Anopheles gambiae larvae by aquatic predators in Western Kenya highlands," Parasites and Vectors, vol. 4, article 128, 2011.

[30] C. L. Lyons, M. Coetzee, and S. L. Chown, "Stable and fluctuating temperature effects on the development rate and survival of two malaria vectors, Anopheles arabiensis and Anopheles funestus," Parasites and Vectors, vol. 6, article 104, 2013.

[31] M. N. Bayoh and S. W. Lindsay, “Temperature-related duration of aquatic stages of the Afrotropical malaria vector mosquito Anopheles gambiae in the laboratory," Medical and Veterinary Entomology, vol. 18, no. 2, pp. 174-179, 2004.

[32] T. M. Lunde, M. N. Bayoh, and B. Lindtjørn, "How malaria models relate temperature to malaria transmission," Parasites and Vectors, vol. 6, article 20, 2013.

[33] A. W. R. McCrae, "Oviposition by African malaria vector mosquitoes. I. Temporal activity patterns of caged, wild-caught, freshwater Anopheles gambiae Giles sensu lato," Annals of Tropical Medicine and Parasitology, vol. 77, no. 6, pp. 615-625, 1983.

[34] E. Rejmankova, A. Harbin-Ireland, and M. Lege, "Bacterial abundance in larval habitats of four species of Anopheles (Diptera: Culicidae) in Belize, Central America," Journal of Vector Ecology, vol. 25, pp. 229-239, 2000.

[35] M. G. Kaufman, E. Wanja, S. Maknojia, M. N. Bayoh, J. M. Vulule, and E. D. Walker, "Importance of algal biomass to growth and development of Anopheles gambiae larvae," Journal of Medical Entomology, vol. 43, no. 4, pp. 669-676, 2006.

[36] M. Coetzee, M. Craig, and D. le Sueur, "Distribution of African malaria mosquitoes belonging to the Anopheles gambiae complex," Parasitology Today, vol. 16, no. 2, pp. 74-77, 2000.

[37] G. M. Nicholson, "Fighting the global pest problem: preface to the special toxicon issue on insecticidal toxins and their potential for insect pest control," Toxicon, vol. 49, no. 4, pp. 413422, 2007.
[38] F. O. Okumu, S. S. Kiware, S. J. Moore, and G. F. Killeen, "Mathematical evaluation of community level impact of combining bed nets and indoor residual spraying upon malaria transmission in areas where the main vectors are Anopheles arabiensis mosquitoes," Parasites \& Vectors, vol. 6, article 17, 2013.

[39] J. V. Rao and P. Kavitha, "In vitro effects of chlorpyrifos on the acetylcholinesterase activity of euryhaline fish, Oreochromis mossambicus," Zeitschrift fur Naturforschung Section C, vol. 65, no. 3-4, pp. 303-306, 2010.

[40] G. Zhou, N. Minakawa, A. K. Githeko, and G. Yan, "Association between climate variability and malaria epidemics in the East Africian highlands," Proceedings of the National Academy of Sciences of the United States of America, vol. 101, no. 8, pp. 23752380, 2004.

[41] G. Zhou, N. Minakawa, A. K. Githeko, and G. Yan, "Climate variability and malaria epidemics in the highlands of East Africa," Trends in Parasitology, vol. 21, no. 2, pp. 54-56, 2005.

[42] M. Pascual, B. Cazelles, M. J. Bouma, L. F. Chaves, and K. Koelle, "Shifting patterns: malaria dynamics and rainfall variability in an African highland," Proceedings of the Royal Society B: Biological Sciences, vol. 275, no. 1631, pp. 123-132, 2008.

[43] S. I. Hay, G. D. Shanks, D. I. Stern, R. W. Snow, S. E. Randolph, and D. J. Rogers, "Climate variability and malaria epidemics in the highlands of East Africa," Trends in Parasitology, vol. 21, no. 2, pp. 52-53, 2005.

[44] J. A. Omumbo, B. Lyon, S. M. Waweru, S. J. Connor, and M. C. Thomson, "Raised temperatures over the Kericho tea estates: revisiting the climate in the East African highlands malaria debate," Malaria Journal, vol. 10, article 12, 2011.

[45] A. B. B. Wilke, A. de Castro Gomes, D. Natal, and M. T. Marrelli, "Control of vector populations using genetically modified mosquitoes," Revista de Saude Publica, vol. 43, no. 5, pp. 869874, 2009.

[46] A. B. B. Wilke and M. T. Marrelli, "Genetic control of mosquitoes: population suppression strategies," Revista do Instituto de Medicina Tropical de Sao Paulo, vol. 54, no. 5, pp. 287292, 2012.

[47] K. R. N. Ng'Habi, D. Mwasheshi, B. G. J. Knols, and H. M. Ferguson, "Establishment of a self-propagating population of the African malaria vector Anopheles arabiensis under semifield conditions," Malaria Journal, vol. 9, article 356, 2010.

[48] M. Q. Benedict, B. G. J. Knols, H. C. Bossin et al., "Colonisation and mass rearing: learning from others," Malaria Journal, vol. 8, supplement 2, article S4, 2009. 

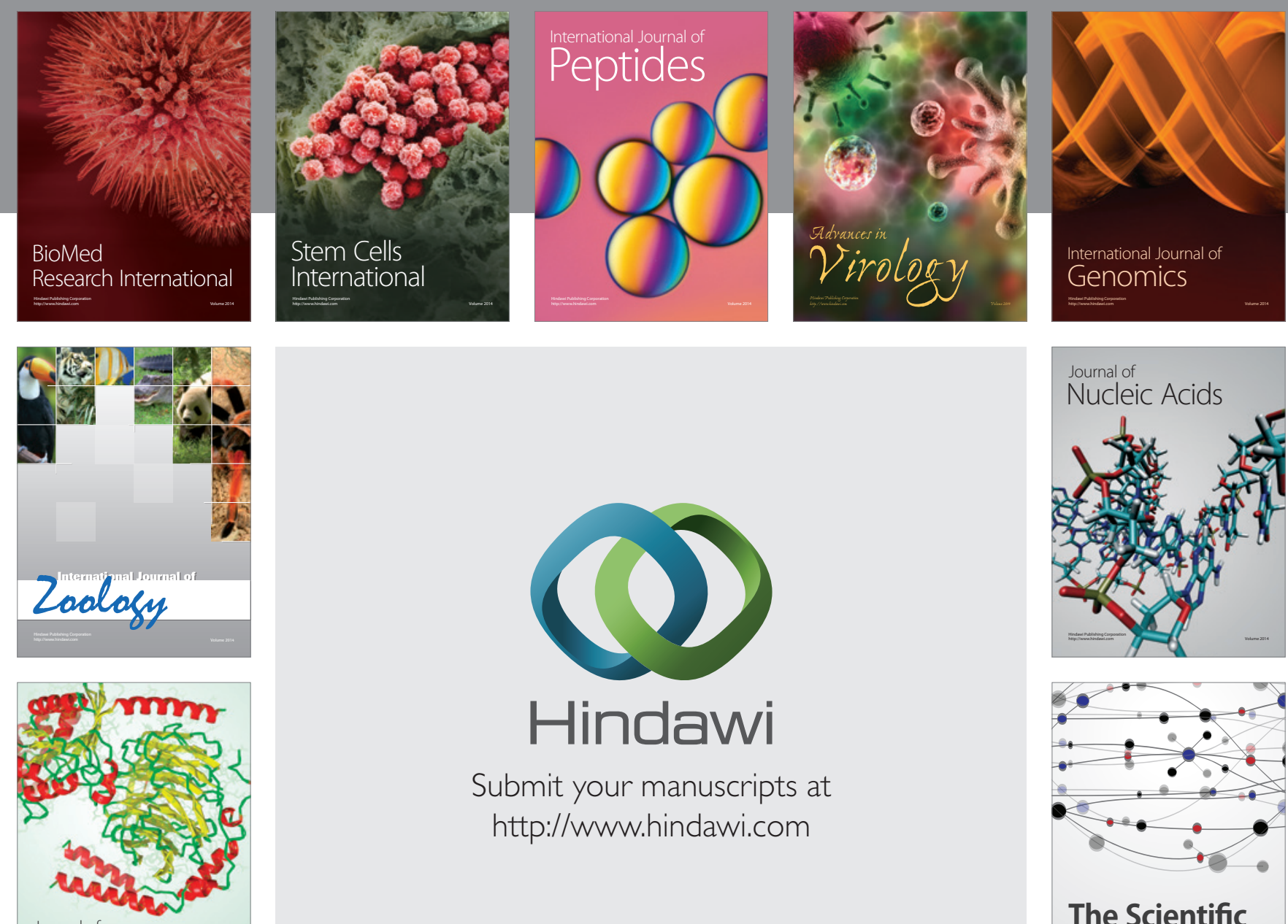

Submit your manuscripts at

http://www.hindawi.com

Journal of
Signal Transduction
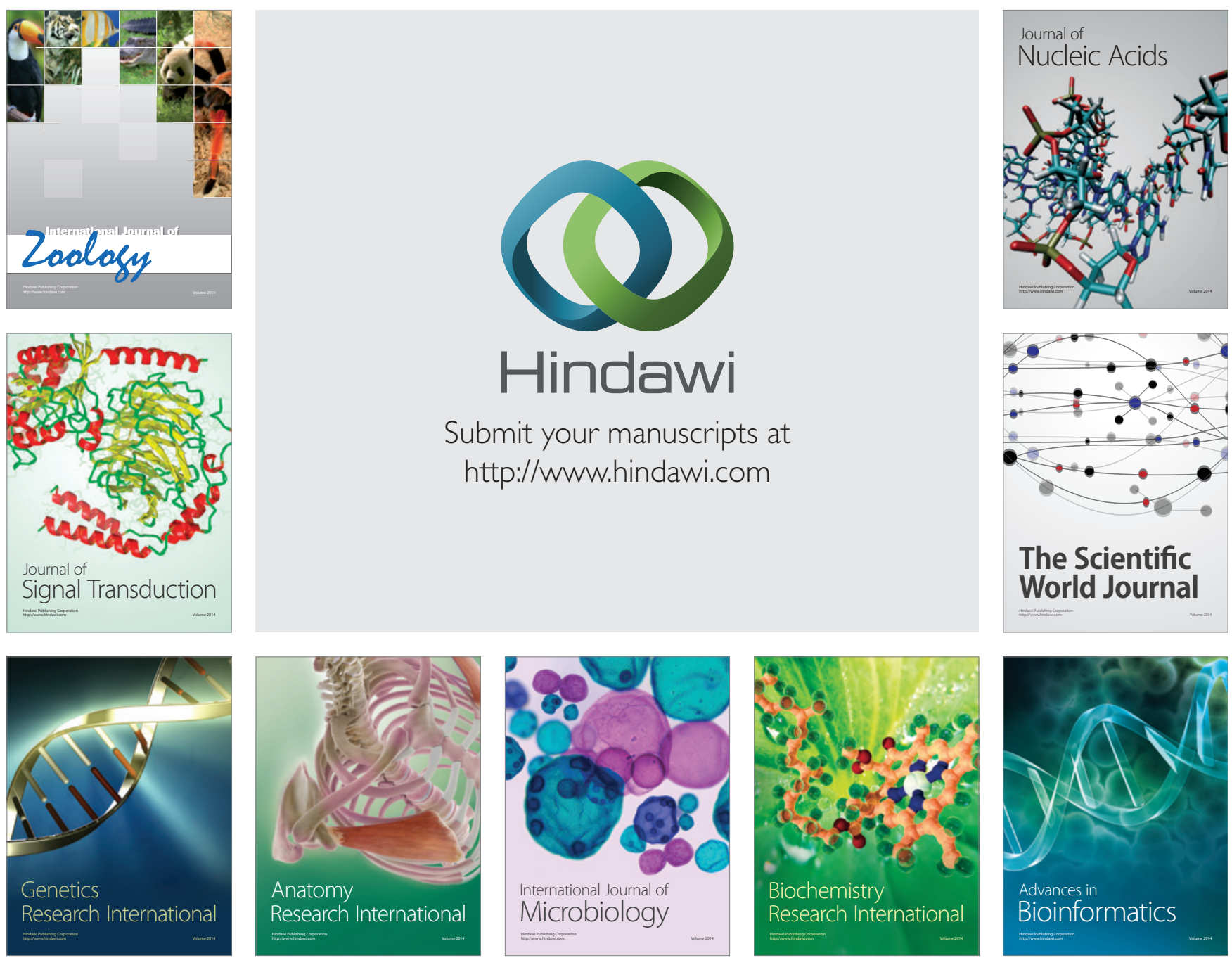

The Scientific World Journal
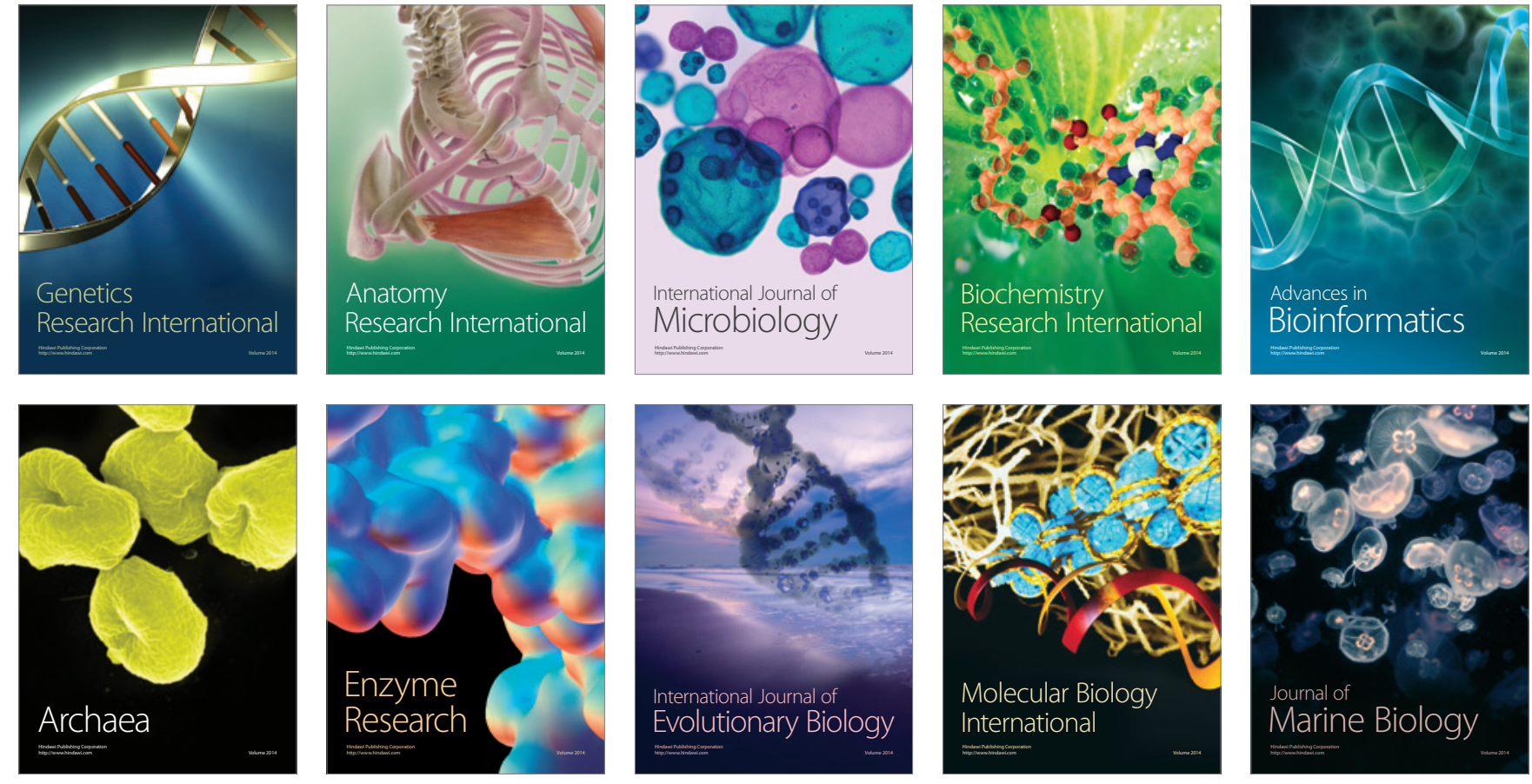